\title{
Dynamic Simulations of Semiconductor Optical Amplifier by Analytical Solution of Traveling-Wave Equation
}

\author{
Khalis.A.Mohammed* Maan M. Shaker* Mozahim I. Azawe** \\ *Technical College of Mosul**Physics Departmen, College of Education, Mosul University
}

\begin{abstract}
Traveling-wave equation of the semiconductor optical amplifier is solved analytically in order to study the dynamical properties of the power output in terms of the input parameters that can influence its operation of semiconductor optical amplifier. Power output (number of photons) was calculated as a function of carrier density, input signal, and bias current. Material gain is found for the amplifier at different wavelengths of the input signal. The model investigated the population inversion along the waveguide of the amplifier. The model, which is used in this article, is one of many mathematical models that can be found in literature . The design of semiconductor amplifier in order to predict the operational characteristics can be obtained from its model. The simplicity of our model is in its ability to incorporate with any external stimulus on the amplifier directly in the wave equation.
\end{abstract}

Keywords: SOA, Traveling-wave equation, Numerical simulations.

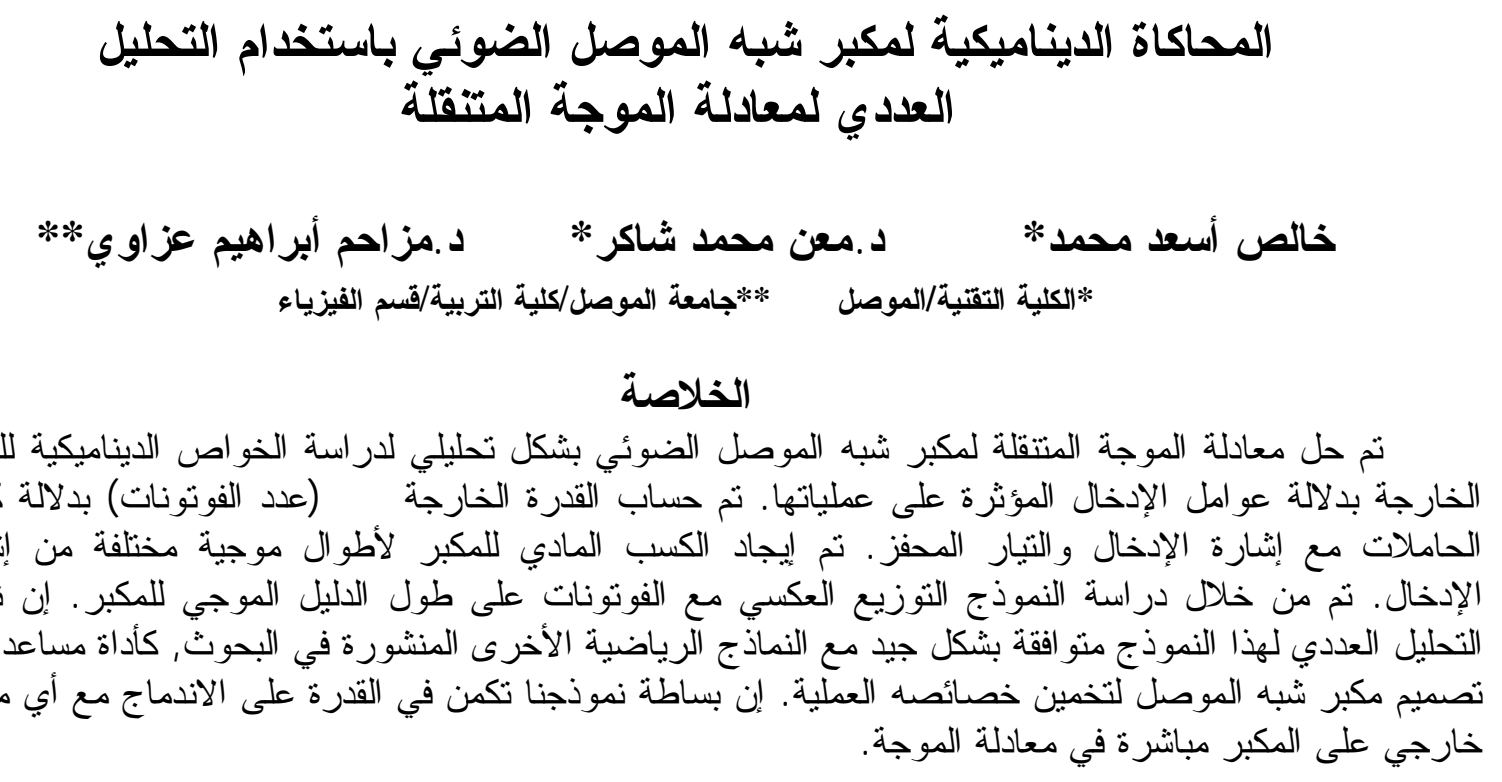

Received 9 Sep. 2007

Accepted 23 Jan. 2008 


\section{INTRODUCTION}

The use of semiconductor optical amplifiers (SOA) is growing in telecom networks. They are faster, cheaper and smaller than doped fiber amplifiers (DFA) for comparable fiber-tofiber gain, and its technology has matured to the point where commercial devices are available for use in optical communication systems[1].

The SOA exhibits non-linear properties and interesting features; one of them is cross gain modulation (XGM), which allows performing all-optical wavelength conversion [2]. The XGM is a novel method for generating ultra-wide band (UWB) monocycle pulses in SOA [3]. A pair of polarity-reversed optical Gaussian pulses was generated at the output of the SOA, to which a Gaussian pulse pump and a continuous-wave probe were applied, and monocycle pulse with a full width at half-maximum of 48 ps and a fractional bandwidth of $188 \%$ was generated at the output of a high-speed photodetector [3].

A multistage interconnection network is determined by the maximum number of internal switching nodes based on commercial SOA switches with polarization-dependent gain [4]. The dependence on both of the packet wavelength and the state of polarization can have a dramatic effect on the optical signal-to-noise ratio degradation due to accumulated amplified spontaneous emission noise with the number of nodes. The model of the polarization-dependent gain saturation in strained bulk SOA has been published before [5], assuming that the polarized optical field can be decomposed into transverse electric and transverse magnetic components that have indirect interaction with each other via the gain saturation. On the other hand, long-wavelength vertical-cavity SOA's (VCSOAs) have many benefits such as high fiber coupling efficiency, polarization-independent gain, the potential to fabricate two-dimensional arrays, and the ability to test devices on wafer [6]. For an overview on the properties of VCSOA as well as emerging applications of this new class of devices, see Ref. $[7,8]$. For the benefit of using SOA, a table given below illustrates the trend in SOA in optical communications [9].

Table 1-1 Major Progress of Optical Communications

\begin{tabular}{|l|l|l|l|}
\hline Year & \multicolumn{1}{|c|}{ Bit Rate } & \multicolumn{1}{|c|}{ Repeater Spacing } & \multicolumn{1}{c|}{ Major Technologies } \\
1980 & $45 \mathrm{Mb} / \mathrm{s}$ & $10 \mathrm{~km}$ & $\begin{array}{l}\text {-. } \lambda=0.8 \mu \mathrm{m} \\
\text {-. Multi-mode fiber } \\
\text {-. GaAs LED }\end{array}$ \\
\hline 1987 & $1.7 \mathrm{~Gb} / \mathrm{s}$ & $50 \mathrm{~km}$ & $\begin{array}{l}\text {-. } \lambda=1.3 \mu \mathrm{m} \\
\text {-. Single-mode fiber } \\
\text {-. InGaAsP Laser Diode }\end{array}$ \\
\hline 1990 & $1.7 \mathrm{~Gb} / \mathrm{s}$ & $60 \sim 70 \mathrm{~km}$ & $\begin{array}{l}\text {-. } \lambda=1.55 \mu \mathrm{m} \\
- \text { Dispersion shifted fiber }\end{array}$ \\
\hline 1996 & $5 \mathrm{~Gb} / \mathrm{s}$ & $\begin{array}{l}\text { Optical Amplifier Spacing } \\
33 \sim 82 \mathrm{~km}\end{array}$ & $\begin{array}{l}\text {-. } \lambda=1.55 \mu \mathrm{m} \\
- \text { Optical Amplifier } \\
\text {-.WDM }\end{array}$ \\
\hline
\end{tabular}

In this paper, numerical simulations based on the traveling-wave and carrier rate equations are carried out by finding the analytical solutions of these equations independently. Material gain and photon density are the most influenced parameters of the SOA that are normally found in any research activity for the dynamics. 


\section{THEORY OF DYNAMICS OF SOA}

SOA is usually modeled by two equations, carrier density $n(z, t)$ and signal field $E_{s k}(z)$, as follows [1]:

$$
\frac{d n(z)}{d t}=\frac{I}{e V}-R(n(z))-\frac{\Gamma}{d W}\left\{\sum_{k=1}^{N_{o}} g_{m}\left(v_{k}, n(z)\right) N_{s k}(z)\right\}
$$

Neglecting the amplified spontaneous emission (ASE) term in the above equation, since the interest is only in the radiative recombination of carriers due to amplified signal, then the signal field is given by [1]:

$$
\frac{d E_{s k}(z)}{d z}=\left[-j \beta_{k}+\frac{1}{2}\left(\Gamma g_{m}\left(v_{k}, n\right)-\alpha_{k}(n)\right)\right] E_{s k}
$$

where $I$ is the pumping current to the SOA, $e$ is the electronic charge, $V$ is active region volume, $v_{k}$ is the signal optical frequency of the mode $k, W$ is the width of the active region of the SOA, $\Gamma$ is the confinement factor, and $N_{s k}$ is the carrier density of the input signal. The $k$-th signal propagation coefficient is given by [1]:

$$
\beta_{k}=\frac{2 \pi n_{e q} v_{k}}{c}
$$

with $\mathrm{c}$ is the speed of light, $n_{e q}$ is the equivalent index of the amplifier waveguide.

$R(n(z))$ represents the term of recombination rate (radiative and nonradiative). The material gain given by the term $g_{m}\left(v_{k}, n\right)$ is calculated from the following equation [1]:

$$
g_{m}\left(v_{k}, n\right)=\frac{c^{2}}{4 \sqrt{2} \pi^{3 / 2} n_{1}^{2} \tau v^{2}}\left(\frac{2 m_{c} m_{h h}}{\hbar\left(m_{c}+m_{h h}\right.}\right)^{\frac{3}{2}} x \sqrt{v-\frac{E_{g}(n)}{h}}\left(f_{c}(v)-f_{v}(v)\right)
$$

The bandgap energy is expressed as:

$E_{g}(n)=E_{g o}-\Delta E_{g}(n)$

Where $f_{c}(v)$ and $f_{v}(v)$ are the probability of both the conduction and the valance band.

The first term of Eqn.(5) is the bandgap energy with no injected carriers, while the second term involves the bandgap shrinkage due to the injected carrier density.

$\alpha_{k}(n)$ is the material loss coefficient and is modeled as a linear function of carrier density [2]:

$\alpha(n)=\mathrm{K}_{o}+\Gamma \mathrm{K}_{1} n_{o}$

$\mathrm{K}_{o}$ and $\mathrm{K}_{1}$ are the carrier-independent and carrier-dependent absorption loss coefficients, respectively. $\mathrm{K}_{o}$ represents the intrinsic material loss. $\mathrm{K}_{1}$ is mainly due to intervalence band absorption. $\Gamma$ is the confinement factor of the dominant factor. 
Equations 1 and 2 are the basic dynamic model and can be solved numerically for defined boundary conditions using the usual Runge-Kutta method. Instead, it is easily to find an analytical solution for the above equations by the Matlab equation solver. The physical parameters of the above equations used in this modeling are taken from Table 1 of Ref. [1]. The two ordinary differential equations can have nontrivial solutions, i.e., a characteristic equation.

\section{NUMERICAL RESULTS}

The material gain of the SOA has to be found firstly in order to proceed further to the above equations of both the carrier density and signal field. To carry out good and fast computations, the gain is often approximated by a polynomial that is a function of photon wavelength and carrier density [1]. This argument is fulfilled during the calculation of the material gain, and typical results are shown in Fig.(1). The gain peak of the SOA $(1.55 \mu \mathrm{m}$ InP-In1-xGaxAsyP1-y ) was around its wavelength and varied due to the variation of the injected carriers. To assess our calculations based on the proposed model, it has to be considered that the material gain is the key issue of any model. As the figure suggests, the overall material gain value is in a good agreement with other model published recently [10].

The carrier density given by Eqn.(1), dependent on the distance measured from the input facet, is said to be a spatial distribution in the amplifier. The equation represents (the first term on the RHS) the addition of carriers to the active region from the bias current, the second term is recombination rate, and the third term is the amplified signal. To get the spatial dependence of carriers, the analytical solution is subjected to an iteration method, after defining the initial conditions at $(\mathrm{t}=0)$, i.e., the carrier density, material gain, and signal input. For a stable solution of the numerical results, the time step chosen should be an order of magnitude less than the carrier lifetime.

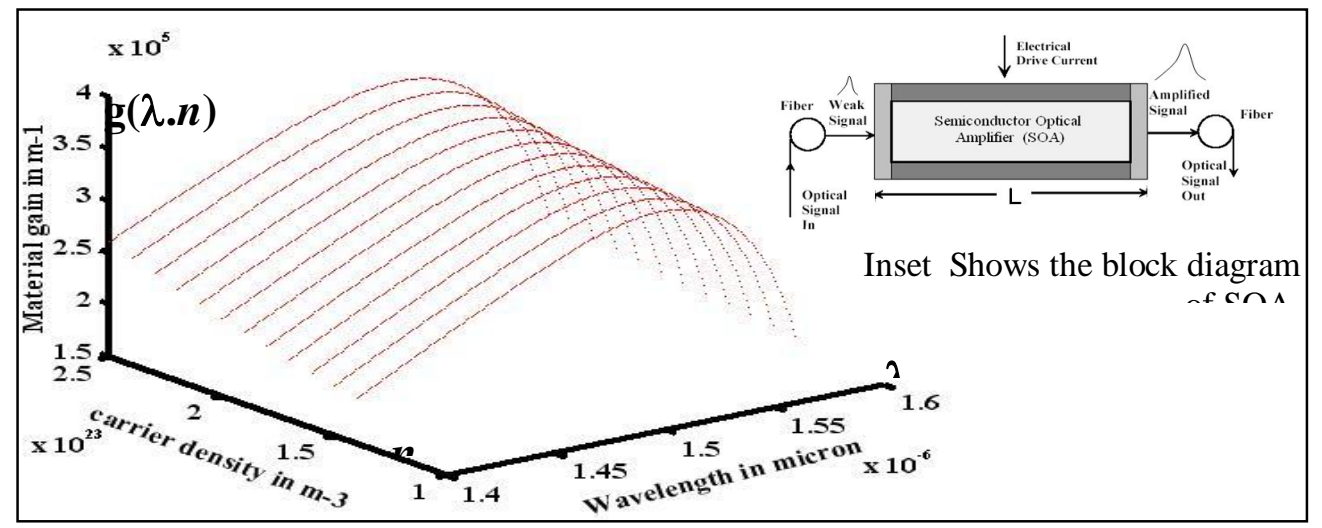

Fig.(1). Typical material gain of the $1.55 \mu \mathrm{m}$ InP-In1-xGaxAsyP1-y SOA plotted as a function of wavelength and carrier density. The device temperature was taken as 300K.

Fig.(2) shows the variation of carrier density with respect to position (means spatial variation related to the amplifier cavity) at different bias currents. The carrier density has asymmetrical spatial distribution, peaking towards the input facet and tailing off towards the output facet. This asymmetry in the distribution is due to the high input power, which has been used in the simulation, is dominating the amplified spontaneous emission. The change in the bias current has increased the carrier depletion rather than having an influence on the peak position. 


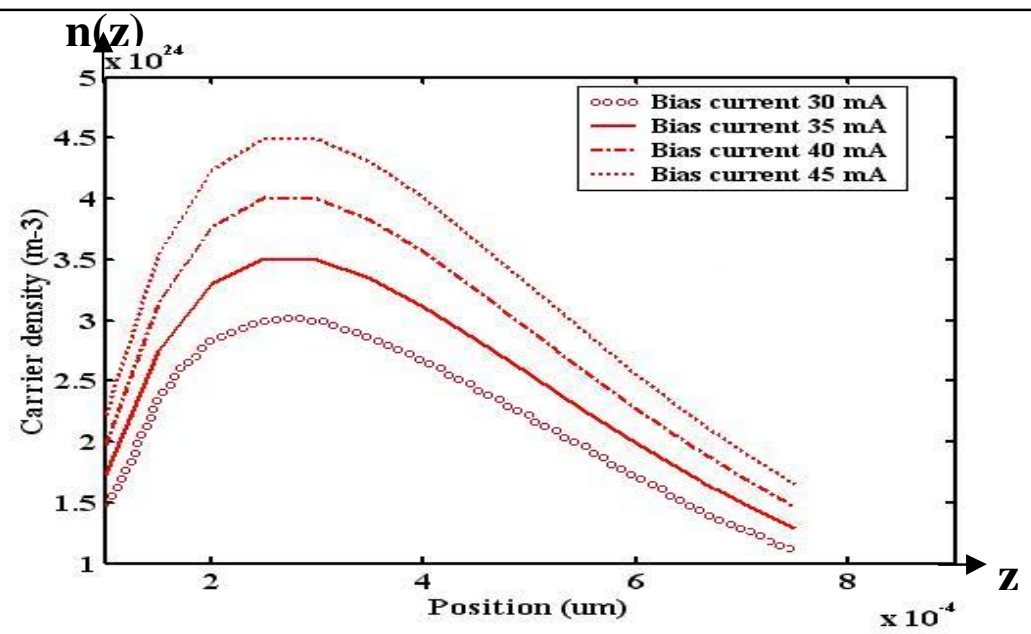

Fig.(2). Spatial distribution of carrier density as a function of biasing current. Input power, and wavelength were kept constant at $-10 \mathrm{~dB}$ and $1.55 \mu \mathrm{m}$, respectively, during the simulations.

Distance is the measured value of axis variation from the input facet rather than the position of spatial variable, which is defined as $\mathrm{z}$ in the paper.

To declare the effect of the input signal power on the output signal photon rate inside the SOA, i.e., spatially variation, we have been able to assess the variation as shown in Fig.(3). The figure illustrates that the power output has its maximum value at the output facet and increases dramatically with input power. The exponential build-up of the photon rate with distance can be explained as an amplification of the signal with positive feedback. In the simulation, the influence of parameters that never be taken into consideration on dynamical properties of the SOA. Nevertheless, these calculations are mainly based on the basic dynamic model of the SOA but with different approach, the analytical solutions. For more inspections on the dynamical stability and noise figure, one has to take into account the influence of parasitic parameters of the SOA. For the time being, focus on the most effective variables on the carrier depletion and power output are very important in the optical communication network.

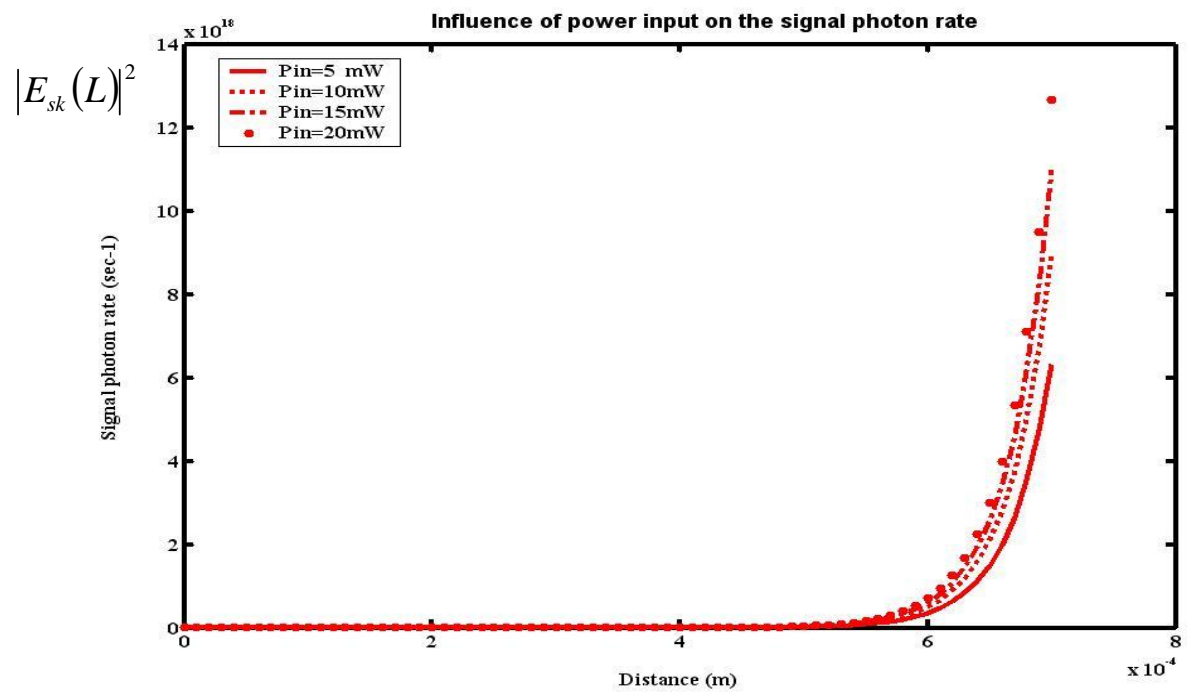

L-with respect to SOA length from input facet

Fig.(3). Variation of the photon rate (power output) with distance measured from the input facet at different input powers. The other parameters were all kept constant, temperature, wavelength, bias current. 
The effect of pumping (biasing) current on the power output as a function of time is illustrated in Fig.(4). The time taken for the carriers to build-up in the upper state needed for population inversion was in the order of magnitude of carrier lifetime. The increase in the photon output rate is obvious from the figure. In order to assess the obtained results, it is clear to say that the exponential increase in the inversion can be deduced from the rate equation. It is worth to notice that, at $(\mathrm{t}=0)$, there are some number of photons due to the input signal. As mentioned before, the analytical solutions for the rate equations are the key to find new effects on the dynamics of the SOA. One of these effects is how carriers, the bias current (see Ref.[11]), will act upon the incident signal, and to what extent of the length of the SOA, inside the active region measured from the front facet. The numerical calculations based on the proposed model were solely dependent on the device given by Ref.[1].

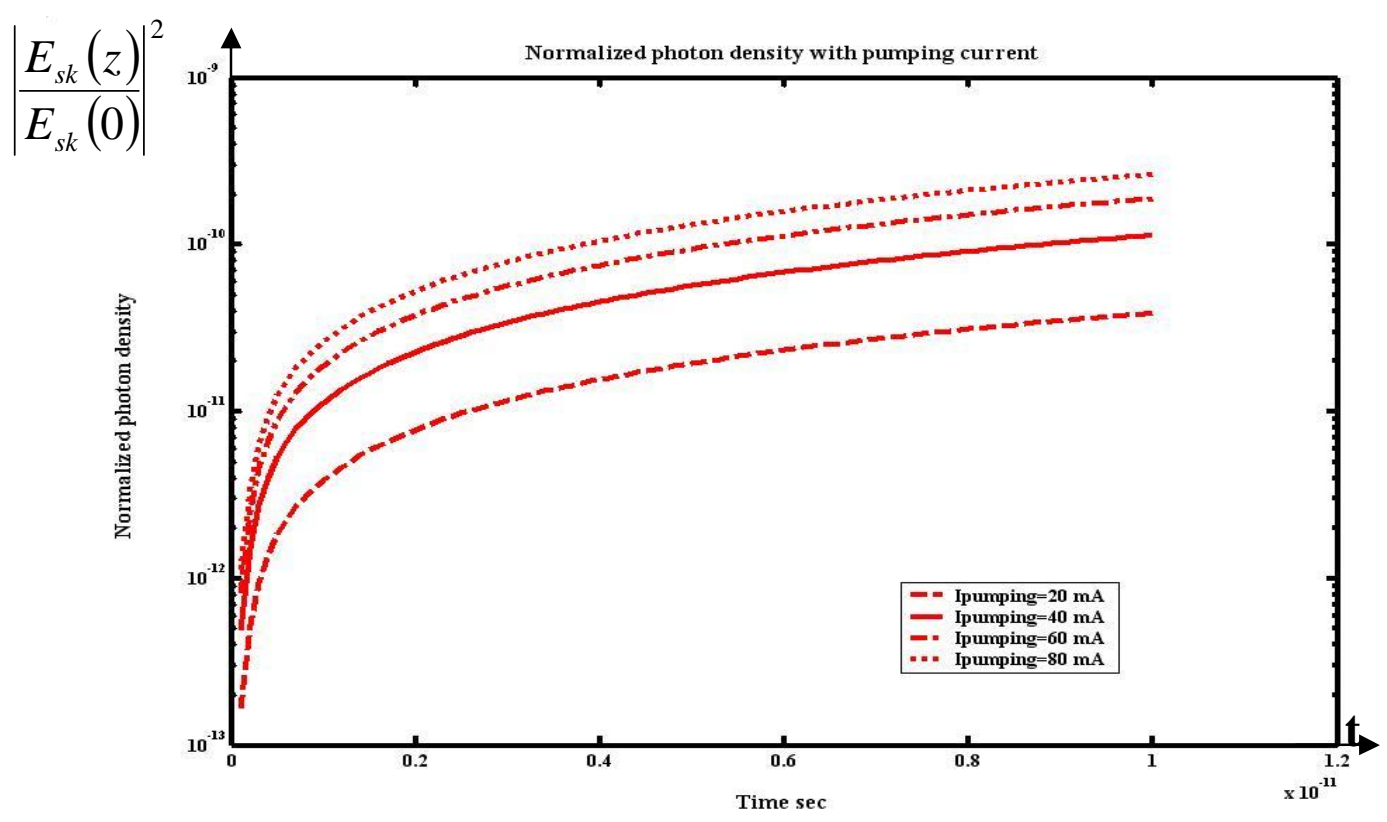

Fig.(4). Typical results of the dependence of the photon output rate on time for inversion as a function of pumping current. All of the rest parameters were kept constant in the calculations.

\section{CONCLUSIONS}

Analytical solutions for the wave equation of the SOA performed using the Matlab package for the sake of obtaining different explanations of the dynamical properties. The output power had been related to the input parameters that can be easily interpreted for any influence on its dynamics. In order to pursue the goal of this investigation, first is to establish an approach that has never been used. This approach will lead us to study the number of photons as a function of input carriers, i.e., the current. It is found that, the carrier density was dependent on the distance from the input facet, giving a spatial distribution inside the SOA cavity. In addition, it is found that, carriers to build-up in the upper state needed for population inversion was in the order of magnitude of carrier lifetime.

Material gain was found for the amplifier at different wavelengths of the input signal. The model studied the population inversion along the waveguide of the amplifier. Numerically, the proposed model was in good agreement with other mathematical models, present in the literature, that are required to aid the design of the semiconductor amplifier in 
order to predict their operational characteristics. The simplicity of the proposed model is the ability to incorporate any external stimulus on the amplifier directly in the wave equation.

\section{REFERENCES}

[1] M. J. Connelly, IEEE J. QUANT. ELECT., QE- 37, 439(2001).

[2] M. J. Connelly, Semiconductor Optical Amplifier, Kluwer Academic, The Netherlands, 2002.

[3]Q. Wang, F. Zeng, S. Blais, and J. Yao, Opt. Lett. 31, 3083(2006).

[4]O. Liboiron-Ladouceur, K. Bergman, M. Boroditsky, and M. Brodsky, IEEE PHOTONICS TECHNOLOGY LETTERS, 18, 1548(2006).

[5]H. J. S. Dorren, D. Lenstra, Y. Liu, Martin T. Hill, and G. Khoe, IEEE J. QUANT. ELECT., QE- 39, 141 (2003).

[6]E. S. Björlin, T. Kimura, and J. E. Bowers, IEEE J. Selected Topics Quant. Elect., 9, 1374(2003).

[7]M.Sanchez, P. Wena, M. Gross, O. Kibar, and S.C. Esener, Proc. SPIE 4649, 96(2002).

[8]E. S. Björlin, SPIE conference on Semiconductor Lasers and Optical Amplifiers for Lightwave Communication Systems, Boston, MA, Aug. 2002.

[9]J-H. Lee, Analysis and Characterization of Fiber Nonlinearities with Deterministic and Stochastic Signal Sources, PhD Thesis, University of Virginia, 2000.

[10]Q. Chen et al., IEEE Photon. Techno. Lett, 16, 1438 (2004).

[11]P. Morel et al., Opt. \& Quant. Electron. 38, 231 (2006). 